\title{
ESTUDO DE VIABILIDADE ECONÔMICA NA CULTURA DA NOZ-MACADÂMIA NO BRASIL ${ }^{1}$
}

\author{
LEONARDO DUARTE PIMENTEL ${ }^{2}$, CARLOS EDUARDO MAGALHÃES DOS SANTOS ${ }^{3}$, AMÉRICO WAGNER \\ JÚNIOR ${ }^{4}$, VERÔNICA AMORIM SILVA ${ }^{5}$, CLAUDIO HORST BRUCKNER ${ }^{6}$
}

RESUMO - A cultura da macadâmia destaca-se como atividade promissora com vistas ao mercado externo. O Brasil está em $6^{\circ}$ lugar na produção mundial, com cerca de 3\% da produção. Há uma demanda crescente pelo produto, o que vem atraindo a atenção de investidores neste ramo do agronegócio. Um dos gargalos da macadamicultura é o elevado período de retorno do capital, sendo importante para o desenvolvimento da atividade o conhecimento do custo de produção e da lucratividade do investimento. Objetivouse, neste trabalho, fazer um levantamento da situação da cultura no Brasil e da viabilidade econômica da atividade. Foi realizada uma estimativa dos custos de produção entre os principais produtores e exportadores do País. Levou-se em consideração os indicadores econômicos: Valor Presente Líquido (VPL), Taxa Interna de Retorno (TIR), Pay-back e Pay-back descontado. Os resultados indicam que a cultura da macadâmia é uma atividade viável e que o sucesso do investimento depende da qualidade do produto.

Termos para indexação: Macadamia integrifolia, agronegócio, custo de produção, exportação, lucratividade.

\section{STUDY OF ECONOMICAL VIABILITY OF THE CULTURE OF MACADAMIA NUT IN BRAZIL}

\begin{abstract}
The macadamia culture stands out as promising activity to the external market. Brazil is in the 6th place in the world production, with approximately $3 \%$ of the production. There is a growing demand for the product, the one that comes attracting the investors' attention in this segment of the agribusiness. One of the difficulties of the macadamia culture is the high period of return of the capital, being important for the development of the activity the knowledge of the production cost and of the profitability of the investment. It was aimed in this work to do a rising of the situation of the culture in Brazil and of the economical viability of the activity. An estimate of the production costs was accomplished among the main producers and exporters of the country. It was taken in consideration the economical indicators: Net Present Value (NPV), Internal Rate of Return (IRR), Pay-back and discounted Pay-back. The results indicate that the culture of macadamia is a viable activity and that the success of the investment depends on the quality of the product.
\end{abstract}

Index Terms: Macadamia integrifolia, agribusiness, production cost, exportation, profitability.

\section{INTRODUÇÃO}

A demanda mundial pelo produto macadâmia vem crescendo em ritmo acelerado em virtude das ações de marketing desenvolvidas pela Australian Macadamia Society (AMS, 2005). Historicamente, o mercado não está sujeito às grandes explosões de safra, característicos das commodities, devido ao tempo de formação do pomar. No Brasil, ainda é pouco conhecida, mas no mercado internacional é bastante apreciada, sendo os EUA o principal consumidor (53\%), seguido pela Austrália (18\%), Ásia (15\%), Europa (8\%) e outros (6\%) (World Horticultural Trade \& U.S. Export Opportunities, 2005).

A nogueira macadâmia é uma espécie subtropical originária da Austrália. O gênero Macadamia pertence à família botânica Proteaceae. A maioria das cultivares é da espécie M. integrifolia, e um menor número de $M$. tetraphylla e de híbridos interespecíficos (Cereda \& Marchi, 1991). A espécie $M$. integrifolia representa $90 \%$ da produção mundial e possui noz de melhor sabor, enquanto a M. tetraphylla é mais utilizada como porta-enxerto. A adaptação à região Sudeste brasileira se deve, provavelmente, às condições climáticas locais serem semelhantes às da região de origem da espécie, uma vez que estão numa mesma faixa de latitude sul. Como referência, pode-se considerar que as exigências climáticas da macadâmia, são, até certo ponto, semelhantes àquelas de culturas consolidadas no País, como o café e citros (Ojima et al., 2005). No Brasil, são aproximadamente 6.000 ha plantados, sendo que, em 2005, a produção foi 3.200 toneladas de noz em casca. Os principais Estados produtores são: São Paulo (33\%), Espírito Santo (31\%), Bahia (18\%) e Rio de Janeiro (10\%) (Sobierajski et al., 2006).

Como a cultura é relativamente recente no País, estudos e dados técnicos são escassos. Poucas instituições têm investido em pesquisas nesta área, e uma das pioneiras é o Instituto Agronômico (IAC), que desenvolve tecnologias para produção e seleção de material adaptado desde 1948 (Ojima et al., 2006). Mas ainda há carência de informações, como produtividade e retorno

\footnotetext{
(Trabalho 197-06). Recebido em: 29-11-2006. Aceito para pulibcação em : 20-07-2007.

${ }^{2}$ Eng. Agr. Msc.Doutorando em Fitotecnia - UFV. Viçosa - MG. CEP 36570-000. Bolsista CNPq. e-mail: agropimentel@yahoo.com.br.

${ }^{3}$ Eng. Agr. MSc. Fitotecnia, Doutorando Genética e Melhoramento - UFV. Bolsista CNPq. e-mail: eduardomagsantos@yahoo.com.br.

${ }^{4}$ Eng. Agr. MSc., D.S Fitotecnia - UFV. Viçosa - MG. CEP 36570-000. Bolsista CAPES. e-mail: americowagner@hotmail.com.

${ }^{5}$ Economista Doméstica, Mestranda em Economia Doméstica, UFV. Viçosa - MG. CEP 36570-000. e-mail: veriamorim@yahoo.com.br.

${ }^{6}$ Eng. Agr. DS., Professor Titular - Departamento de Fitotecnia, UFV. Viçosa - MG. CEP 36570-000. e-mail: bruckner@ufv.br.
} 
econômico, dificultando uma análise mais precisa da lucratividade da cultura.

Em relação à produtividade, os dados encontrados na literatura são divergentes. Dierberger \& Marino Netto (1985) consideraram uma produtividade média anual de noz seca em casca de $75 \mathrm{~kg} /$ planta aos 12 anos (planta adulta), ou $26.800 \mathrm{~kg} /$ ha. Toledo Piza \& Almeida Neto (1991) estimaram produção entre 30 e $40 \mathrm{~kg} / \mathrm{planta}$, equivalendo a 7.500 a $10.000 \mathrm{~kg} / \mathrm{ha}$, respectivamente. Já Simão (1998) refere-se a produções de 30 a $125 \mathrm{~kg} /$ planta, sem comentar a densidade por ha.

Neste contexto, o objetivo deste trabalho foi realizar um levantamento da situação da cultura da noz-macadâmia no Brasil e avaliar a viabilidade econômica da atividade. Consideraram-se a produtividade média alcançada pelos produtores, o custo de produção, os preços do produto e dos fatores de produção, o fluxo de caixa e indicadores econômicos.

\section{MATERIAL E MÉTODOS}

O estudo foi realizado no período de abril a outubro de 2005, a partir de levantamento acerca da cultura da macadâmia em todo o Brasil. Foram consultados na literatura todos os aspectos da cadeia produtiva da cultura, desde dados técnicos, como tratos culturais, variedades, produção, dentre outros, até os aspectos relativos à comercialização do produto, como regiões produtoras, perfil dos produtores, preços nacionais e internacionais, além das tendências de mercado. A maior parte das informações foi obtida por meio de entrevistas com produtores e indústrias do setor, como a Queen Nut-SP, e Coopemac - ES/BA, que também possuem produção própria. Além disso, foram realizadas consultas a pesquisadores, em todo o País, a fim de proceder uma validação ao estudo realizado. Desse modo, puderam-se levantar os dados a seguir comentados.

\section{Manejo dado à cultura pelos principais produtores}

$\mathrm{Na}$ produção brasileira de macadâmia destinada à exportação, tem-se produção agrícola empresarial, com base tecnológica elevada. Assim, utilizou-se modelo semelhante, estimando-se os custos do plantio em propriedade de 100 ha, com aração, gradagem e calagem total; a adubação foi considerada conforme Souza et al. (1999), para fertilidade natural baixa do solo. Não se considerou o uso de irrigação, pois a maioria dos pomares de São Paulo, para onde foi elaborado o estudo, não são irrigados. Como premissas, foram adotados: espaçamento 8,0 x 6,0 m (208 plantas/ha); Latossolo (textura média); replantio de mudas (5\%); adubação/calagem e tratos fitossanitários conforme indicados para a cultura; produtividade média esperada de $4.160 \mathrm{~kg} / \mathrm{ha}$ de noz seca em casca ( $10 \%$ umidade), sem carpelo na idade adulta; taxa de retorno em amêndoa (TR $)^{1}$ média esperada de $25 \%$.

\section{Custos de produção}

Os custos de produção foram divididos em 7 partes: Operações Mecanizadas (A); Operações Manuais (B); Insumos (C); Custos Administrativos (D), Benfeitorias e Ferramentas (E);
Máquinas e Implementos (F), e Terra (G). Os custos de produção foram calculados do seguinte modo:

\section{A) Operações Mecanizadas:}

$\mathrm{O}$ valor das operações mecanizadas de aração, gradagem e terraciamento foi considerado com base no valor médio do aluguel, uma vez que essas máquinas e equipamentos só são utilizados na implantação da cultura. Para as demais, considerouse a utilização de máquinas e implementos próprios. Os componentes do custo podem ser considerados em Custos Fixos (depreciação e juros) e Custos Variáveis (combustível, lubrificantes, manutenção e mão-de-obra). Porém, para este trabalho, consideramos apenas os custos variáveis, sem a mãode-obra (calculada separadamente no item 'B'), uma vez que os custos fixos foram contemplados no fluxo de caixa. Exemplo de cálculo do custo horário, considerando conjunto trator $(75 \mathrm{cv})$ com carreta (4 ton), é apresentado no Tabela 1.

\section{B) Operações Manuais:}

Para cálculo do item 'B', foram considerados trabalhadores fixos e contratados (temporários). Fixos foram: 1 tratorista e 2 braçais, com custo mensal de $\mathrm{R} \$ 600,00+87 \%$ de encargos sociais, e R $\$ 400,00+87 \%$ (cada braçal), respectivamente. Convertendose esses custos em reais por ha/ ano ( $\mathrm{R} \$ /$ ha ano), tem-se $\mathrm{R} \$ 134,64$ e R $\$ 89,76$. Para mão-de-obra contratada, considerou-se o valor diário de $\mathrm{R} \$ 15,00+87 \%$ de encargos por braçal e $\mathrm{R} \$ 80,00+87 \%$ para técnicos, o que equivale a $\mathrm{R} \$ 28,05$ e $\mathrm{R} \$ 149,60$, respectivamente (Tabela 2).

\section{C) Insumos:}

Calculou-se o preço médio entre os principais distribuidores, exceto para o preço das mudas de macadâmia, que foi considerado o valor de venda da Queen Nut / Dois CórregosSP.

\section{D) Custos administrativos:}

Foram considerados os gastos com mão-de-obra administrativa, viagens, contabilidade, luz/telefone, conforme estimativa de FNP Consultoria e Agroinformativos (2005) para citros, em módulo de 100 ha.

\section{E) Benfeitorias e Ferramentas:}

Estimaram-se os gastos com a construção de um galpão $\left(300 \mathrm{~m}^{2}\right)$ para secagem e armazenamento, equipamentos de escritório (computadores, móveis, aparelhos de fax, etc.), além de ferramentas básicas, como carrinhos de mão, enxadas, dentre outros.

\section{F) Máquinas e Implementos:}

Consideraram-se as compras de 1 veículo (camioneta), 1 trator $75 \mathrm{cv}, 1$ roçadeira hidráulica, 1 carreta (4 toneladas), 1 pulverizador de barras (herbicida), 1 atomizador e 1 descarpelador. Os dois últimos só entraram no fluxo de caixa no ano 5 , quando 
inicia a produção, e os demais, na implantação do pomar. Adotaram-se 25 anos como período de vida útil dessas máquinas e implementos. Os custos foram obtidos a partir do valor médio do custo de máquinas fornecido por FNP Consultoria e Agroinformativos (2005).

G) Terra:

Utilizou-se como custo de oportunidade da terra o valor de arrendamento para a região de Jaú-SP (região produtora de macadâmia). Segundo o IEA (2005), nessa região, o valor anual médio do arrendamento de terras, para o ano de 2005 , foi de R\$ 433,00/ha.

\section{Fluxo de caixa do investimento}

Na planilha do fluxo de caixa, estimaram-se as receitas e despesas durante o horizonte do projeto, que é de 50 anos (vida útil das plantas). Todos os valores foram expressos em reais por hectare ( $\mathrm{R} \$ / \mathrm{ha})$. Os custos com máquinas e equipamentos foram inseridos apenas no primeiro e quinto anos, de acordo com a necessidade de compra, e no vigésimo sexto ano, quando se torna necessária a substituição. Considerou-se que a produtividade média $(\mathrm{kg} / \mathrm{ha})$ é crescente a partir do $5^{\circ}$ ano e estabilizada a partir do $12^{\circ}$ ano, quando a cultura atinge a fase adulta. A partir do início da produção, levou-se em consideração a tributação referente apenas ao imposto de renda gerado pela atividade, uma vez que não há incidência do ICMS (Imposto Sobre Circulação de Mercadorias e Serviços) nos produtos agrícolas destinados à exportação. Para avaliar a rentabilidade da cultura, a partir do fluxo de caixa, foram elaborados os seguintes indicadores econômicos: TIR (taxa interna de retorno); VPL (valor presente líquido); Pay-back (tempo de retorno do capital), e Pay-back descontado (tempo de retorno do capital incluindo juros), e para o cálculo desses indicadores, foi utilizado o aplicativo computacional EXCEL versão 2002. Todos os valores são referentes ao período de outubro de 2005, quando o preço pago ao produtor por $\mathrm{kg}$ de noz seca em casca (sem carpelo) foi em média US $\$ 1,40^{2}$, para produto com TR de $25 \%$ (taxa de retorno em amêndoa), sendo esta TR a adotada no fluxo de caixa.

\section{RESULTADOS E DISCUSSÃO}

\section{Produtividade, produtores e comercialização}

Em pesquisa realizada com diversos dos principais produtores brasileiros e com técnicos e pesquisadores do setor, verificou-se que a produtividade média alcançada é de $20 \mathrm{~kg}$ de noz seca em casca (sem carpelo) aos 12 anos (densidade de 208 plantas/ha), chegando a aproximadamente 4,2 ton/ha (Figura 1). Essa produtividade está abaixo daquelas encontradas na literatura (Dierberger \& Marino Netto 1985; Simão 1998; Toledo Piza \& Almeida Neto 1991). Porém, segundo Squinca et al. (2003) e Sobierajski et al. (2006), a produtividade média dos produtores paulistas pode variar entre $20 \mathrm{~kg}$ e $30 \mathrm{~kg}$ de nozes em casca (sem carpelo) por árvore adulta, dependendo da variedade e dos tratos culturais. Na Austrália são encontradas árvores centenárias em plena produção, em função da rusticidade e do vigor dessa espécie. No entanto, os pomares brasileiros mais antigos foram implantados em meados da década de 60 (Dierberger \& Marino Netto, 1985) e, desse modo, consideramos a vida útil, para fins de estudo de viabilidade, de 50 anos. Em relação à produção, esses autores sugerem uma produção crescente ao longo dos anos sob condições experimentais, porém consideramos uma produtividade estável a partir do $12^{\circ}$ ano, uma vez que não se têm dados acerca de pomares comerciais no Brasil a partir dessa idade.

Os produtores de macadâmia podem ser classificados como pequenos de 1 a 5 ha; médios de 5,1 a 50 ha e grandes, acima de 50 ha (CATI, 2005). A maior parte da produção brasileira vem dos médios e grandes produtores, sendo que, com poucas exceções, os grandes são as próprias indústrias do setor.

Normalmente, a produção é vendida para beneficiadoras após secagem. As beneficiadoras pagam pelo produto de acordo com TR, que é a relação entre o peso das nozes e o peso das amêndoas resgatadas (Squinca et al., 2003). A fase de pós-colheita da macadâmia é de extrema importância, pois influencia diretamente na qualidade (percentagem de amêndoas inteiras, teor de óleo e açúcar) do produto e, conseqüentemente, na TR, que definirá o preço no mercado, que é cotado em dólar. No Brasil, essa taxa fica, em média, em $23 \%$. No entanto, esse valor segue uma escala exponencial. Para se ter idéia, o produto com TR de $20 \%$ é vendido por US\$ 0,96 ; TR $25 \%$ por US $\$ 1,40$, e TR $30 \%$ por US $\$ 2,01$. Em estudo de competição entre a noz-macadâmia paulista e a noz norte-americana, Martin (1992) conclui que somente os produtores que obtêm rendimento acima de $4.000 \mathrm{~kg} / \mathrm{ha}$, e um produto de qualidade intermediária, conseguem rentabilidade financeira acima de $20 \%$. Entende-se por qualidade intermediária TR acima de $23 \%$. Desse modo, pode-se inferir que a qualidade (TR) é um fator decisivo na viabilidade econômica dessa atividade.

As principais empresas exportadoras no Brasil são a Coopemac (ES/BA), responsável por cerca de $40 \%$ das exportações nacionais, e a Queen Nut (SP), com 35\%. Outras, como a Tropical Passas (SP/RJ), também compram o produto, porém em menor quantidade.

Em relação ao mercado nacional, tem ocorrido, nos últimos anos, uma demanda crescente pelo produto, sendo que as empresas do ramo têm incentivado o cultivo e estão pagando ágio pela fidelidade e pela quantidade aos seus fornecedores, além de elas próprias estarem ampliando seus pomares. Em relação ao mercado internacional, as exportações vêm aumentando de maneira constante ao longo dos últimos anos (World Horticultural Trade \& U.S. Export Opportunities, 2005), o que reflete a crescente demanda pelo produto nos principais mercados consumidores.

\section{Custos de produção}

Os itens de maior peso na implantação do pomar ( $1^{\circ}$ ano $)$ foram os insumos e o maquinário, com custo médio de $\mathrm{R} \$ 1.631,34$ / ha e R $\$ 1.008,20 /$ ha, respectivamente (Tabela 2). Para insumos, o subitem de maior peso foi o custo com mudas ( $\mathrm{R} \$ 1.220,80 / \mathrm{ha}$ ), correspondendo a $74 \%$ do gasto com insumos. Para o maquinário, o subitem de maior peso foi o trator ( $\mathrm{R} \$ 670,00 / \mathrm{ha})$, 
correspondendo a $66 \%$ do gasto. Na seqüência, os itens de maior impacto no custo de produção foram: mão-de-obra administrativa, operações mecanizadas, operações manuais, custo de oportunidade da terra e benfeitorias.

Considerando o somatório dos custos de produção até o primeiro ano de receitas positivas $\left(6^{\circ}\right.$ ano), tem-se um desembolso total de $\mathrm{R} \$ 14.130,66 /$ ha (Figura 2). Pode-se observar que o item de maior peso foram operações manuais, que, por sua vez, é influenciado pela mão-de-obra temporária, tanto nas operações de plantio quanto na colheita. Squinca et al. (2003), em amostragem das propriedades paulistas, estimaram as operações mecanizadas como item de maior peso, provavelmente pelo fato de incluir operações mecanizadas e custo com maquinário juntos, diferindo do presente estudo, em que se optou por analisar esses fatores separados.

Benfeitorias e ferramentas foi o item de menor peso no custo total. Mesmo assim, o subitem de maior peso foi o galpão para armazenamento e secagem, que só entrou no fluxo de caixa no $5^{\circ}$ ano (início da produção comercial).

\section{Viabilidade do investimento:}

O fluxo de caixa (Tabela 3 ) foi realizado com valores reais ao longo de todo o horizonte do investimento. Para analisar a lucratividade da cultura, foram calculados os indicadores econômicos VPL, TIR, Pay-back e Pay-back descontado, uma vez que esses indicadores econômicos são tomados como padrão nas análises de investimentos a longo prazo.

O VPL compara todas as entradas e saídas de dinheiro na data inicial do projeto, descontando todos os valores futuros do fluxo de caixa a determinada taxa de juros pre-estabelecida, que mede o custo de capital. Basicamente, é o cálculo de quanto os futuros pagamentos somados estariam valendo no presente (Afonso Júnior et al., 2006). Neste investimento, considerou-se o valor dos juros a taxa $\mathrm{SELIC}^{3}$ deflacionada, que foi de $14 \%$ no ano (BACEN, 2006). É sabido que, na análise de viabilidade de investimentos agrícolas, é comum adotar juros subsidiados, porém utilizamos aqui a taxa SELIC a fim de permitir comparações com outros setores, uma vez que o capital investido é muito alto e, assim, pode-se optar por outros investimentos. O VPL (14\%) calculado foi de $\mathrm{R} \$ 16.789,47 / \mathrm{ha}$ (Tabela 4). Porém, podem-se obter valores maiores, por exemplo, trabalhando com juros menores e/ ou reduzindo os custos de produção. Nos 6 primeiros anos, os itens de maior impacto no VPL foram as operações manuais, insumos e custo de oportunidade da terra (Figura 2), respectivamente, uma vez que o capital investido foi o de maior peso e deve ser desembolsado nos períodos iniciais, quando a cultura não apresenta receita.

Ainda de acordo com Afonso Júnior et al. (2006), a TIR é a taxa de juros que anula o VPL, isto é, que torna $\mathrm{VPL}=0$. Essa taxa independe da taxa de juros do mercado financeiro, sendo, portanto, uma taxa intrínseca do investimento analisado, dependente apenas do fluxo de caixa. No estudo em questão, obteve-se TIR de 26,05\%. Pode-se considerar que a TIR representa uma rentabilidade limítrofe entre a viabilidade e a inviabilidade do investimento, sendo um indicador econômico que deve ser analisado junto ao VPL para prover maior confiabilidade e visibilidade ao retorno do capital investido. Desse modo, pode-se inferir que, para as condições preestabelecidas, há um ganho real de $12,05 \%$ quando descontada a taxa anual de juros $(14 \%)$ empregada no cálculo do VPL, evidenciando a viabilidade do investimento.

Em relação ao Pay-back e ao Pay-back descontado, o capital seria recuperado com 9 e 11 anos, respectivamente. O elevado tempo de retorno do capital pode ser considerado como um dos fatores limitantes da macadamicultura no Brasil. Neste sentido, tornam-se necessárias pesquisas na área de manejo da cultura (densidade, poda, adubação, pragas e doenças), adaptando os tratos culturais às necessidades ambientais brasileiras (Sobierajski et al., 2005), favorecendo maiores produtividades e TR, conseqüentemente menor tempo de retorno do capital investido.

Para reduzir o tempo de retorno do capital, há duas maneiras: aumento na produtividade e TR da cultura, e/ou realizar plantio consorciado/intercalar. No primeiro caso, por ser um fator intrínseco da espécie, depende mais de investimentos em pesquisas. No caso do consórcio, este já vem sendo a principal estratégia dos produtores brasileiros para formação de pomares de macadâmia. Apesar de plantios intercalares serem bastante comuns no período de formação da cultura, neste estudo, não foram considerados, a fim de não interferirem no fluxo de caixa do investimento e, assim, ocultar o retorno real da cultura. De acordo com Bruckner \& Martinez (1991), podem ser realizados plantios de macadâmia consorciados com mamão, maracujá, cacau, café e algumas culturas anuais. Neste caso, fruteiras de ciclo curto, como mamão e maracujá, são mais interessantes, pois proporcionam geração de renda mais rapidamente.

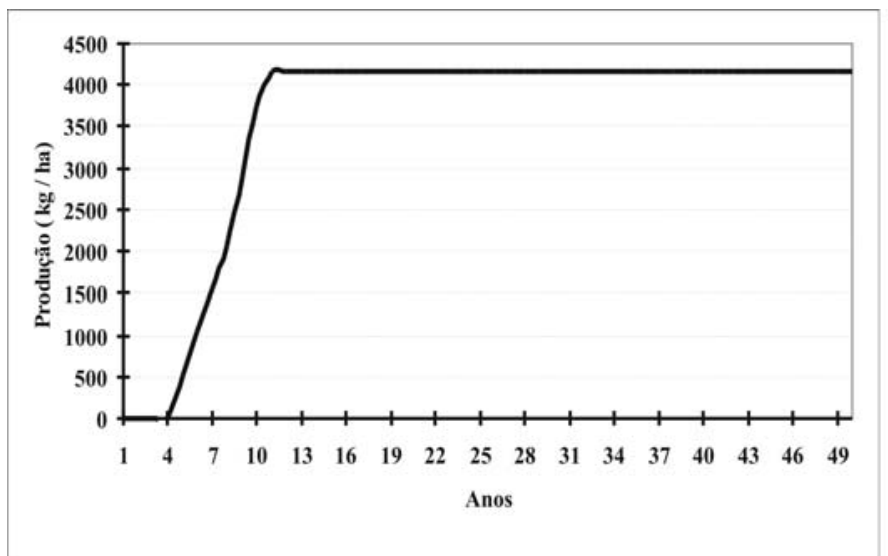

FIGURA 1 - Estimativa da produtividade da macadâmia $(\mathrm{kg} / \mathrm{ha})$, densidade 208 plantas por ha (espaçamento de $8 \times 6 \mathrm{~m}$ ) em latossolo de textura média, na região Sudeste do Brasil.

\footnotetext{
3 Taxa SELIC (Sistema Especial de Liquidação e Custódia) no ano de 2005 ficou em torno de 19,6\%; e, neste trabalho, considerou-se a taxa SELIC deflacionada, $14 \%$.
} 


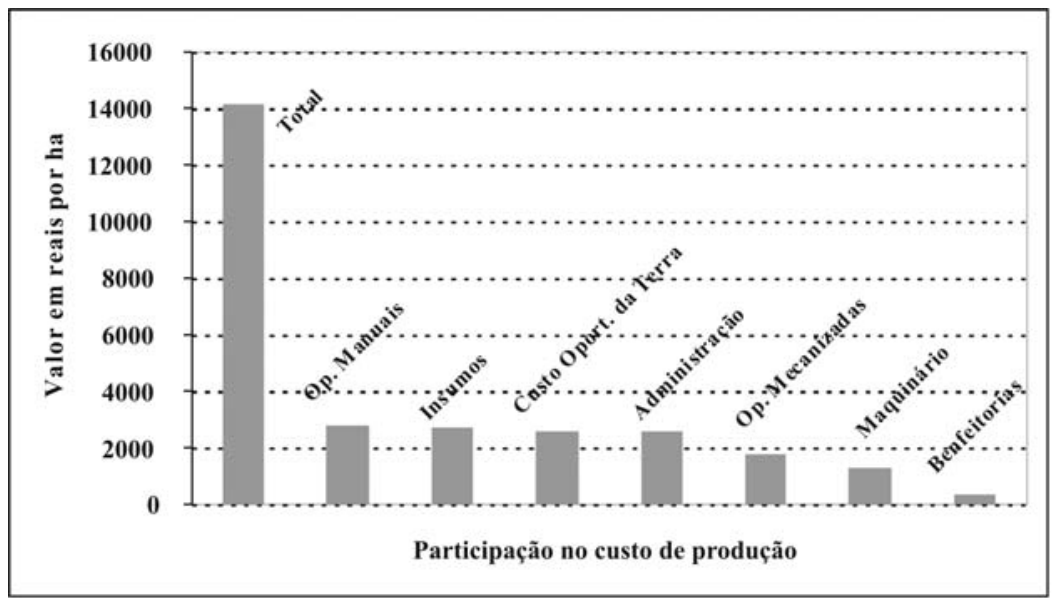

FIGURA 2 - Desembolso de caixa da cultura da macadâmia até o $6^{\circ}$ ano do investimento (primeiro ano de receita positiva), considerando o somatório de cada item no custo total (R\$14.130,66/ha), para o Estado de São Paulo.

TABELA 1- Exemplo de custo horário, referente ao conjunto trator e carreta (HM Tp $75 \mathrm{cv}+$ carreta 4 toneladas) na cultura da macadâmia, Estado de São Paulo, em reais de out. 2005.

A1) Custo horário do trator:

Consumo de combustível:

Consumo $\left(\mathrm{C}_{\mathrm{L} / \text { hora }}\right)=7$ litros e preço do óleo diesel $\mathrm{R} \$ 1,84 / \mathrm{L}$.

Custo com combustível: $\mathrm{CC}_{\mathrm{RS} h o r a}=7 \times 1,84$

Lubrificantes (Graxa e Óleo):

Preço do kg graxa: R \$ 6,00 e consumo de 0,05 kg/hora.

Custo com graxa: $\mathrm{CG}_{\mathrm{RS} \text { hora }}=0,05 \times 6$

Preço do Litro de óleo: R R 7,00 e consumo de 0,048L/hora.

Custo com óleo: $\mathrm{CO}_{\mathrm{RShora}}=0,048 \times 7$

Manutenção: $100 \%$ do valor de compra $(\mathrm{Vc})$ durante toda a vida útil $(\mathrm{Vu})$ :

Taxa de manutenção $\left(\mathrm{TM}_{\text {anual }}\right)=100 \% / \mathrm{Vu}$

Custo de manutenção: $\mathrm{CM}_{\mathrm{R} \$ \text { /ano }}=67000$ x 0,04

Considerando 1.000 horas de uso anual, tem-se:

$$
\mathrm{CC}_{\mathrm{RSh \text {hora }}}=12,88
$$

$\mathrm{CG}_{\mathrm{R} \$ \text { hora }}=0,30$

$\mathrm{CO}_{\mathrm{RS} / \text { hora }}=0,33$

$\mathrm{TM}_{\text {anua }}=100 / 25=4 \%$ a.a.

$\mathrm{CM}_{\mathrm{RS} / \mathrm{ano}}=2680,00$

$\mathrm{CM}_{\mathrm{RS} \text { hora }}=2,68$

A2) Custo horário da carreta:

Taxa de manutenção $\left(\mathrm{TM}_{\text {anual }}\right)=5 \%$ a.a.

$\mathrm{TM}_{\mathrm{R} / \text { ano }}=1,05 \times \mathrm{Vc} \quad \mathrm{TM}_{\mathrm{R} / \text { ano }}=0,05 \times 3700$

$\mathrm{TM}_{\mathrm{RS} / \text { ano }}=185,00$

Considerando 200 horas de uso anual, tem-se:

$\mathrm{CM}_{\mathrm{RS} \text { hora }}=0,92$

A3) Custo total conjunto trator/carreta:

Custo total do trator $\left(\mathrm{CC}_{\mathrm{RShora}}+\mathrm{CG}_{\mathrm{RShora}}+\mathrm{CO}_{\mathrm{RSh} \text { hora }}+\mathrm{M}_{\mathrm{RS} \text { hora }}\right)=\mathrm{R} \$ 16,19 /$ hora.

Custo total da carreta $\left(\mathrm{M}_{\mathrm{R} \$ \text { hora }}\right)=\mathrm{R} \$ 0,92 /$ hora.

Custo total do conjunto trator/carreta $=\mathrm{R} \$ 17,11 /$ hora $($ Tabela 1$)$. 
TABELA 2 - Coeficientes técnicos e estimativa de custo de produção, para a cultura da noz- macadâmia no Estado de São Paulo, densidade de 208 plantas/ha, produtividade média de $4160 \mathrm{~kg} /$ ha a partir do $12^{\circ}$ ano, em reais, de out. 2005, por ha.

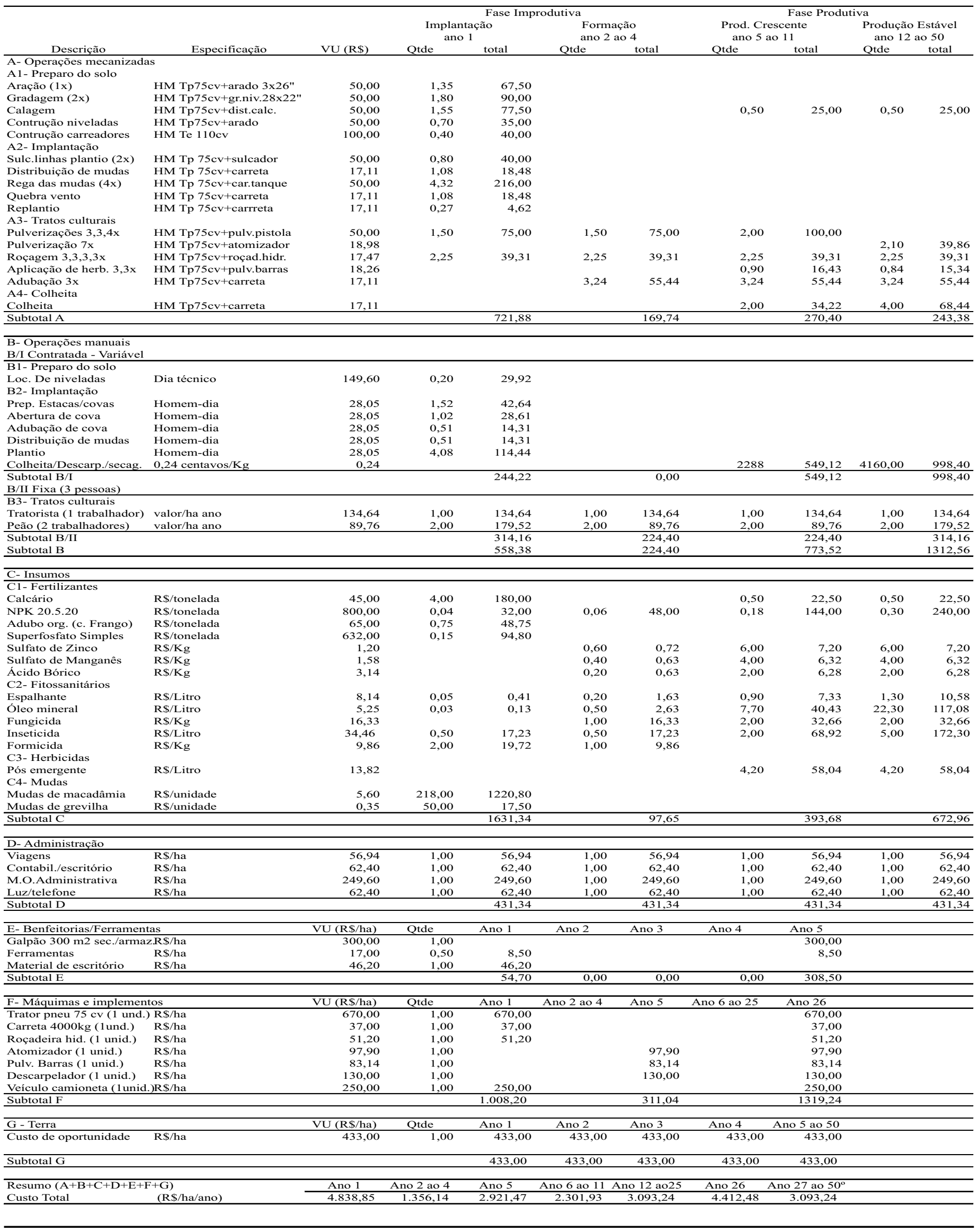

Rev. Bras. Frutic., Jaboticabal - SP, v. 29, n. 3, p. 500-507, Dezembro 2007 
TABELA 3 - Fluxo de caixa da noz-macadâmia no Estado de São Paulo, densidade de 208 plantas/ha, produtividade média de $4.160 \mathrm{~kg} /$ ha a partir do $12^{\circ}$ ano, em reais de out. 2005, por ha.

\begin{tabular}{|c|c|c|c|c|c|c|c|c|c|c|c|c|c|c|c|c|}
\hline \multirow{3}{*}{ Descrição/Custos } & \multicolumn{16}{|c|}{ Fases da cultura } \\
\hline & \multirow{2}{*}{$\begin{array}{l}\text { Plantio } \\
\text { Ano } 1\end{array}$} & \multirow{2}{*}{$\begin{array}{c}\text { Formação } \\
\text { Ano } 2\end{array}$} & \multirow[b]{2}{*}{ Ano 3} & \multicolumn{4}{|c|}{ Produção Crescente } & \multicolumn{9}{|c|}{ Produção Estável } \\
\hline & & & & Ano 4 & Ano 5 & Ano 6 & Ano 7 & Ano 8 & Ano 9 & Ano 10 & Ano 11 & Ano 12 & Ano 13 & Ano 14 & Ano 15 & 16 a $050^{(2)}$ \\
\hline Op. Mecanizadas & 721,88 & 169,74 & 169,74 & 169,74 & 270,40 & 270,40 & 270,40 & 270,40 & 270,40 & 270,40 & 270,40 & 243,38 & 243,38 & 243,38 & 243,38 & 243,38 \\
\hline Op. Manuais & 558,38 & 224,40 & 224,40 & 224,40 & 773,52 & 773,52 & 773,52 & 773,52 & 773,52 & 773,52 & 773,52 & $1.312,56$ & $1.312,56$ & $1.312,56$ & $1.312,56$ & $1.312,56$ \\
\hline Insumos & $1.631,34$ & 97,65 & 97,65 & 97,65 & 393,68 & 393,68 & 393,68 & 393,68 & 393,68 & 393,68 & 393,68 & 672,96 & 672,96 & 672,96 & 672,96 & 672,96 \\
\hline Administração & 431,34 & 431,34 & 431,34 & 431,34 & 431,34 & 431,34 & 431,34 & 431,34 & 431,34 & 431,34 & 431,34 & 431,34 & 431,34 & 431,34 & 431,34 & 431,34 \\
\hline Benfeitorias & 54,70 & - & - & - & 308,50 & - & - & - & - & - & - & - & - & - & - & - \\
\hline Maquinário & $1.008,20$ & - & - & - & 311,04 & - & - & - & - & - & - & - & - & - & - & - \\
\hline Custo Oport. da Terra & 433,00 & 433,00 & 433,00 & 433,00 & 433,00 & 433,00 & 433,00 & 433,00 & 433,00 & 433,00 & 433,00 & 433,00 & 433,00 & 433,00 & 433,00 & 433,00 \\
\hline Tributação & - & - & - & - & 172,67 & 303,82 & 469,56 & 518,46 & 745,28 & 984,64 & 831,26 & 831,26 & 831,26 & 831,26 & 831,26 & 831,26 \\
\hline Custo Total/ha & $4.838,85$ & $1.356,14$ & $1.356,14$ & $1.356,14$ & $3.094,14$ & $2.605,75$ & $2.771,49$ & $2.820,39$ & $3.047,21$ & $3.286,57$ & $3.133,19$ & $3.924,50$ & $3.924,50$ & $3.924,50$ & $3.924,50$ & $3.924,50$ \\
\hline Produtividade $\mathrm{Kg} / \mathrm{ha}$ & - & - & - & - & 520 & 1.040 & 1.560 & 2.080 & 2.912 & 3.744 & 4.160 & 4.160 & 4.160 & 4.160 & 4.160 & 4.160 \\
\hline Receita total / ha ${ }^{(1)}$ & - & - & - & - & $1.645,28$ & $3.290,56$ & $4.935,84$ & $6.581,12$ & $9.213,57$ & $11.846,02$ & $13.162,24$ & $13.162,24$ & $13.162,24$ & $13.162,24$ & $13.162,24$ & $13.162,24$ \\
\hline Lucro Bruto & $(4.838,85)$ & $(1.356,14)$ & $(1.356,14)$ & $(1.356,14)$ & $(1.448,86)$ & 684,81 & $2.164,35$ & $3.760,73$ & $6.166,36$ & $8.559,44$ & $10.029,05$ & $9.237,74$ & $9.237,74$ & $9.237,74$ & $9.237,74$ & $9.237,74$ \\
\hline Lucro Líquido & $(4.838,85)$ & ) $(6.194,98)$ & $(7.551,12)$ & $(8.907,26)$ & $(10.356,12)$ & $(9.671,31)$ & $(7.506,96)$ & $(3.746,24)$ & $2.420,12$ & $10.979,56$ & $21.008,61$ & $30.246,35$ & $39.484,09$ & $48.721,82$ & $57.959,56$ & $67.197,30$ \\
\hline Lucro Bruto Acumulado & $(4.838,85)$ & $(6.028,44)$ & $(7.071,94)$ & $(7.987,30)$ & $(8.845,14)$ & $(8.489,47)$ & $(7.503,42)$ & $(6.000,50)$ & $(3.838,83)$ & $(1.206,73)$ & $1.498,54$ & $3.684,35$ & $5.601,73$ & $7.283,64$ & $8.759,00$ & $10.053,17$ \\
\hline
\end{tabular}

TABELA 4 - Indicadores de viabilidade do investimento na cultura da noz- macadâmia no Estado de São Paulo, densidade de 208 plantas/ha, produtividade média de $4.160 \mathrm{~kg} / \mathrm{ha}$ a partir do $12^{\circ}$ ano, em reais de out. 2005 , por ha.

\begin{tabular}{ll}
\hline Indicador & Valores \\
\hline VPL (14\%) & $16.789,47$ \\
TIR & $26,05 \%$ \\
Pay-Back & 9 anos \\
Pay-Back Descontado & 11 anos \\
\hline
\end{tabular}

\section{CONCLUSÕES}

1- Para as condições estudadas, a cultura da macadâmia é uma atividade viável, apresentando um VPL(14\%) de R\$16.789,47/ ha e TIR de 26,05\%. O período de retorno do capital (Pay-back) foi de 9 anos e 11 anos para o Pay-back descontado, indicando que o tempo de retorno do capital é o principal gargalo da cultura da macadâmia no Brasil.

2- A qualidade do produto (TR - taxa de rendimento em amêndoa) é fator decisivo na formação do preço e, conseqüentemente, na lucratividade do investimento.

\section{REFERÊNCIAS}

AFONSO JÚNIOR, P. C.; OLIVEIRA FILHO, D.; COSTA, D. R. Viabilidade econômica de produção de lenha de eucalipto para secagem de produtos agrícolas. Engenharia Agrícola, Jaboticabal, v. 26, n. 1, p. $28-35,2006$.

AGRIANUAL 2005: anuário da agricultura brasileira. São Paulo: FNP Consultoria \& Agroinformativos. 2005. 500 p.

AMS - AUSTRALIAN MACADAMIA SOCIETY. Macadâmia Statistics. Disponível em: $<$ http://www.macadamias.org $>$. Acesso em: 05 maio. 2005.

BACEN-BANCOCENTRAL DOBRASIL. Relatório Anual 2005. v.41, 225 p. Disponível em: <http://www.bcb.gov.br/htms/ banual2005/rel2005introdp.pdf/>. Acesso em: 10 out. 2006.

BRUCKNER, C. H.; MARTINEZ, H. E. P. Plantio e condução. In: SÃO JOSÉ, A. R. (Ed.). Macadâmia: tecnologia de produção e comercialização. Vitória da Conquista: DFZ/UESB, 1991. p. 95117.

CATI - COORDENADORIA DE ASSISTÊNCIA TÉCNICA INTEGRAL. L.U.P.A. - levantamentos de unidades de produção rural. Disponível em: <http://www.cati.sp.gov.br/novacati/ serviços/mapas/culturas/macadamia>. Acesso em: 8 abr. 2005. 
CEREDA, E.; MARCHI, M. Botânica e caracterização da nogueira macadâmia. In: SÃO JOSÉ, A. R. (Ed.). Macadâmia: tecnologia de produção e comercialização. Vitória da Conquista: DFZ/UESB, 1991. p. 05-28.

DIERBERGER, J. E.; MARINO NETTO, L. Noz Macadâmia, uma nova opção para a fruticultura brasileira. Piracicaba: ESALQ, 1985. p.120.

IEA - INSTITUTO DE ECONOMIA AGRÍCOLA. Valor da terra nua. Disponível em: <http://www.iea.sp.gov.br/>. Acesso em: 04 abr. 2005 .

MARTIN, N. B. Análise do potencial de competição da produção da noz macadâmia em São Paulo e no Havaí. Informações Econômicas, São Paulo, v. 22, n. 10, p. 9-53, 1992.

OJIMA, M.; DALL'ORTO, F. A. C.; BARBOSA, W; RIGITANO, O. Macadamia integrifolia Maid. e Bet. Campinas: Instituto Agronômico de Campinas, Seção de Fruticultura de Clima Temperado, 2005, p.1-2 (Boletim, 200).

OJIMA, M.; DALL'ORTO, F. A. C. eBARBOSA, W. Melhoramento da macadâmia. Disponível em: <http://www.iac.sp.gov.br/>. Acesso em: 10 out. 2006.

SIMÃO, S. Tratado de fruticultura. Piracicaba: Fealq, 1998. p.760.
SOBIERAJSKI, G. da R.; FRANCISCO, V. L. F. dos S.; ROCHA, P.; GHILARDI, A. A.; MAIA, M. L. Noz-Macadâmia: produção, mercado e situação no Estado de São Paulo. Informações Econômicas, São Paulo, v.36, n.5, p. 25-36, 2006.

SOUZA, M. de; GUIMARÃES, P. T. G.; CARVALHO, J. G. de; FRAGOAS, J. C. Macadâmia. In: RIBEIRO, A. C.; GUIMARÃES, P. T. G.; VENEGAS, V. H. A. (Ed.) Recomendações para o uso de corretivos e fertilizantes em Minas Gerais. 5 ed. Viçosa: CFSEMG, 1999. p. 250-252.

SQUINCA, A. F. R.; CAMPOS, E. M.; MARTINS, M. I. E. G. Avaliação econômica da produção e comercialização da nozmacadâmia no Estado de São Paulo. In: CONGRESSO INTER. DE ECONOMIA E GESTÃO DE REDES AGROALIMENTARES, 4., 2003, Ribeirão Preto. Anais... Ribeirão Preto: USP, 2003. p. 1-10.

TOLEDO PIZA, J. de; ALMEIDA NETO. A colheita e o beneficiamento da macadâmia. In: SÃO JOSÉ, A. R. (Ed). Macadâmia: tecnologia de produção e comercialização. Vitória da Conquista: DFZ/UESB, 1991. p. 131-147.

WORLD HORTICULTURAL TRADE \& U.S. EXPORT OPPORTUNITIES. Situation And Outlook For Macadamia Nuts. 2002. Disponível em: $<$ http://www.fas.usda.gov/>. Acesso em: 12 abr. 2005. 